\title{
ET-BE: Rules and Guidelines for Writing a Thesis
}

\section{Purpose}

This document lays down the requirements, options and best practices for ET-BE students writing a BSc or MSc thesis. Doctoral candidates are also recommended to follow these guidelines when writing papers or theses. It comprises several sections which describe the 'rules' (requirements) and some advice for different aspects of a thesis. This document is not a template. The relevant repository ${ }^{1}$ provides several templates that go into the purpose of possible chapters/sections.

Note that the requirements in this document are specific to the ET-BE department and might deviate from rules in other departments, faculties or study programs. It is obligatory that you investigate whether your study program has any specific thesis document requirements (often on a Canvas page). Program specific requirements supersede anything stated in this document. However, if program and department requirements are not in conflict, it is required to incorporate both. Supervisors are allowed to be more strict and specific, but not lenient with respect to the requirements given in this document.

This document was adapted from the work of Prof. $\mathrm{H}$. Vallery [1]. It has been modified and extended by Frank Halfwerk, Venkat Kalpathy Venkiteswan and Arvid Keemink*. Version: May 11, 2021

\section{General Writing Advice}

- Think of your audience: who will read the thesis or paper (e.g. fellow students in the same program)? Explain theory and motivate (design) choices clearly at the level that your audience can follow.

- Start with a logical structure, add text and figures later. You could start with the structures proposed in Sec. III and the templates.

- Have someone review your paper and structure, early in the writing process.

- Polish the abstract and keep it clear and concise, because many readers will read only this.

\section{Thesis STRUCTURE REQUIREMENTS}

Your thesis document requires certain components or chapters. Some of those are required, even when writing a paper. The required structure is as follows, given in required order, where each starts on a separate page:

- Required: Cover page, free choice in design, but must include:

- Document type, one of the following options: Bachelor Thesis, Master Thesis, Bachelor's Thesis or Master's Thesis.

- Student name: first name and last name written in full. E.g.: John F. Doe. Adding your own academic title (e.g. BSc at the end) is optional.

*Corresponding author, e-mail: a.q.1.keemink@utwente.nl. Not for discussion or clarification, that is what your supervisor is for.
- Only if confidential, state CONFIDENTIAL in all caps.

- Thesis title

- Faculty and department: Engineering Technology Biomechanical Engineering

- Graduation committee members ${ }^{\dagger}$, including titles. You can find titles of UT staff on people.utwente. $\mathrm{nl}^{2}$. Be consistent for all members in the type of title use (EU/NL or UK/US).

- ET-BE Report number. This will be provided by the department secretary.

- Month and Year (month in which defense takes place)

- Logo of the University of Twente, and possible other institution(s) at which you do your assignment (MST, Roessingh, etc.).

- Optional: Any (sub)title page(s) can be placed after the cover page, but not directly on the back of the cover page.

- Optional: Acknowledgments

- Optional: Preface

- Required: Abstract. Note that the abstract is not a teaser but a condensed version of the work. See for example [2] for more information on abstract writing.

- Required: Table of Contents (ToC)

- The cover page and the ToC are not shown in the ToC.

- Acknowledgments, Preface and Abstract are shown in the ToC.

- Everything before the 'Introduction' has roman lower case (i, ii, iii, iv) page numbering. The Introduction starts on page 1 , by definition. How to: MS Word ${ }^{3}$, ${ }_{\mathrm{AT}} \mathrm{X}^{4}$. The cover page and its backside are not numbered.

- Optional: List of Abbreviations

- Optional: List of Figures

- Optional: List of Tables

- Optional: List of Symbols

- Choose one of the options below for the remainder of the thesis body:

1) A more traditional thesis. Typical structure could be: Introduction, Background, Methods, Results, Discussion, Conclusion, References, Appendices with references.

2) A report on a design assignment: Problem analysis, Design assignment, Requirements, Concepts and Selection, Prototype description, Discussion, Conclusion, References, Appendices with references.

3) One (or multiple) paper(s). A general introduction and discussion are required, also when the body is comprised of (a) research paper(s). Typical structure: Introduction, Paper(s) incl. references, Discussion, References for the Introduction and Discussion, Appendices with references.

${ }^{\dagger}$ Graduation committee $=$ Examination Committee (NL 'Afstudeercommissie'). Do not confuse with Examination Board (NL 'Examencommissie'). 
The purpose of sections are explained in more detail in the templates on the repository ${ }^{1}$. Note that these proposed structures are recommended, but not set in stone.

- Required: Paper size A4, portrait. Font size of the bodytext is never larger than $11 \mathrm{pt}$. Margins are $2.5 \mathrm{~cm}$ on all sides.

- Required: Digital document version is in PDF in which rasterized figures are reduced to minimally 300 dpi, especially high resolution photos.

\section{Structuring the TeXt}

\section{Required}

- Each paragraph should contain one distinct idea or message. Also, the reader should see the paragraph's topic in either the first, second or last sentence of the paragraph.

- Ensure logical connection within and between paragraphs.

- Readers should know where to find which information, so use mutually exclusive headings that do not repeat in other sections or chapters.

- Do not place chunks of 'loose' text between section heading and subsection heading. One or two sentences are possible, but only for an overview of the entire section.

- Do not skip heading levels. Avoid using more than three levels (paper: section, subsection, subsubsection; or thesis: chapter, section, subsection). If you need to split at a deeper level, revise your structure.

\section{Writing Advice}

- It works well to write down the main ideas first, and only then to expand them to paragraphs. In this way you ensure a logical flow and solid argument between paragraphs, sections and chapters.

- Except for the introduction, in which a paperoutline/thesis-outline might be mentioned, a section (chapter) should not contain only a single subsection (section). They consist of either one whole part, or more than two subsections (sections).

- Balance paragraph length. Instead of fluctuating long and short paragraphs, it is easier for the reader to have medium-sized paragraphs throughout.

- Preferably each chapter in a thesis starts on a new page, which is the odd numbered right page. This can indeed lead to some empty pages.

- Be consistent with use of line breaks between paragraphs. Do not equalize column length in a two-column paper unless a journal requires it.

\section{LANGUAGE}

\section{Required}

- Thesis reports and papers are written in English. Choose, together with your supervisors, UK or US spelling, and stick to a single one throughout the document. Note that old-fashioned UK spelling of certain words is falling out of fashion ('metre' to 'meter').
- Tenses: past tense for actions that have been completed or happened in the past, present tense for (derived) factual statements, and future tense for planned/future work.

- Do not use contractions ('don't') or colloquial speech ('way more', 'a lot of'). Note that 'can't' is rewritten as 'cannot' (no space).

- Do not use qualifiers such as 'very' and 'much'. Also, do not use 'significant' to mean 'large'. Significant has a very precise meaning in academic writing.

- Avoid using qualitative adjectives like 'easy', 'simple', 'difficult', 'straightforward', 'complex', 'trivial', etc. For example, do not write: 'a complex method', 'a complicated procedure', 'it is easy to see'.

\section{Writing Advice}

- Write simple, short and concise sentences. Aim for around 10 words.

- Avoid static verbs and passive voice; try to write in active voice.

- Do not use 'I' or 'One' (this is just an old fashioned form of I) to refer to yourself (do not use, for example, 'One can see from the data') in writing.

- Pronoun 'we' is sometimes used, but is not preferred either. See if you can avoid it without resorting to passive voice.

- Look up the rules for hyphenation in English, particularly for compound modifiers, to know the difference between a 'red haired person' and a 'red-haired person'.

- Look up the rules for 'which' and 'that' and their required punctuation.

- Merriam-Webster ${ }^{5}$ helps with spelling and hyphenation. Do not trust $\mathrm{AT}_{\mathrm{E}} \mathrm{X}$ 's automatic hyphenation and provide it hyphenation exceptions.

- Whenever you look something up (like grammar rules), do not trust newsgroups, forum posts or other such sources. Use reliable sources, such as books or websites by university writing centers.

- Try to use only English words you know, and do not look for synonyms and alternatives. This is technical (academic) writing, not creative writing. Reuse words and reuse sentences for similar statements.

\section{Vi. Citations and Referencing Others}

\section{Required}

- Never use other people's ideas, text or images without proper citation. Also cite, and rephrase your previous work when you reuse it.

- Never copy any piece of text, even with a reference. This is a form of plagiarism. If you want to copy text, it can only done as a proper quote (see Writing Advice below). Literally copying your own previously published text is also plagiarism.

- Provide evidence for each single claim that is not an obvious fact, either in the form of a reference, or by your own experiment, deduction or calculation. Any claims your make and figures you show that have no reference are assumed to be your original work. 
- For all images from others, even public domain ones, check the license or obtain permission for reuse. All non-confidential theses should be published on essay.utwente. $\mathrm{ll}^{6}$. Therefore, all images that you did not make yourself require a license and citation. Publishers will tell you how to write such a citation. They even have an automated process to get permission, for example via RightsLink or Copyright Clearance Center (mostly instantaneous and free for students' theses).

- Cite primary, original literature. Do not cite reviews or textbooks that have compiled the knowledge; go back to the original sources. You can cite a review or book if insights and conclusion from that review or book bring you something that the original work does not.

- You can refer to authors directly, but a rule of thumb is to use no more than two or three authors. So if a paper has eight authors, then preferably say: 'Einstein et al. [2] proved that...', but if the paper has only two authors: 'Einstein and Doe [3] showed...'. Incorrect: 'Einstein and Doe et al.'.

- You can also refer to the work directly, example: 'In [3] an overview is given...', but do not start a sentence with a numerical reference. Other citation styles work the same: 'In (Einstein, 1917) ...', but such citation styles also allow for the following form: 'According to Einstein (1917) ...'. In $\mathrm{AT}_{\mathrm{E}} \mathrm{X}$ the natbib package has two citation options.

- Use a reference manager (e.g. Mendeley ${ }^{7}$ or EndNote (University Supported)). These can be exported to several formats to work with LTEXor in MS Word.

- As explained in Sec. III, if you write one or more papers to put in your thesis, then those have their own references within the paper. The thesis Introduction and Discussion then also have their own references. Appendices always have their own references (even if they overlap with the Introduction, Discussion or Papers). If you have a classical thesis, the body and the Appendices each have their own references.

\section{Writing Advice}

- Avoid direct citation or quoting (“...”) whenever possible, instead rephrase statements when citing. If you do use a quote, make this explicit.

- Read until you understand each paper you are citing, or at least the portion of the paper you are referring to. Do not just rely on abstracts or, worse, secondary literature that cites the original source (incorrectly). If you feel it is impossible to read and understand cited references, narrow down the scope of your work until it is.

- For IEEE style referencing, preferably group references (e.g. [1]-[4] to avoid [1,2,3,4] or [1][2][3][4]) with the cite or natbib package in $\mathrm{ET}_{\mathrm{E}} \mathrm{X}$.

- Do not blindly trust reference exports from reference managers. Manually go over the exported data to ensure your reference list has consistent formatting. Common issues: missing author initials, different ways of writing the same journal name, some references that do have urls or dois, while others do not.

\section{MATH \& UNITS}

An overview of mathematical symbols is given in $\mathrm{LT}_{\mathrm{E}} \mathrm{X}^{8}$, or MS Word's modern equation editor. For LTEX you can also draw your symbol ${ }^{10}$, and it will give the code.

\section{Required}

- Define each single variable explicitly. Do not rely on self-explaining variables or indices like $x_{\max }$ or on the reader's background knowledge.

- Operators and common mathematical functions are typeset upright. The only allowed exception is differentiation. Incorrect: $\sin (x), \ln (x), \exp (x)$, correct: $\sin (x), \ln (x), \exp (x), \frac{d x}{d t}, \frac{\mathrm{d} x}{\mathrm{~d} t}$.

- Variables and parameters only have a single symbol, and preferably so do all their sub- and superscripts (exception for upright 'min' or 'max' sub-/superscript).

- Variables and parameters are typeset differently from text and words, typically italicized (for scalars, but sometimes also for vectors or matrices) or upright boldface (for vectors and matrices). For example: $x=y^{2}$ or $\mathbf{A x}=\mathbf{b}$. Preferably typeset scalars, vectors, and matrices differently.

- In the exceptional case that you need words in an equation (if it conveys the message more clearly than substitution by variables), they should be upright and contain spaces, e.g.

$$
\text { grade }=\frac{\text { number of points }}{\text { maximum number of points }} \cdot 9+1 .
$$

- You can use two superscripts and two subscripts: ${ }_{2}^{1} R_{4}^{3}$. Use brackets if indices could be confused with an exponent. But subscripts and superscripts are placed directly on top of each other. Incorrect: $x^{2}{ }_{i}, x_{i}{ }^{2}$, correct: $x_{i}^{2}$.

- Dòts, hâts, tildẽs, bārs, etc. are above the main symbol and are never bold. Incorrect: $x_{\text {long }}, \dot{\mathbf{p}}$, correct: $\dot{x}_{\text {long }}, \dot{\mathbf{p}}$.

- Every physical and non-normalized quantity has units. Mention the units when a numerical value is given or do so 'earlier' while defining the symbols in the case that using an SI [3] unit invalidates the equation.

- It is a common mistake to put square brackets around the unit. This is incorrect according to the SI [3] and the ISO norms [4]. You should use normal brackets to state units for a quantity, the square brackets act as a 'unit of'operator. Example: For a force $F$ with units of Newtons $(\mathrm{N})$; correct: $[F]=\mathrm{N}$, incorrect: $F[\mathrm{~N}]$. For axes labels in plots, use either ' $F(\mathrm{~N})$ ', 'Force $(\mathrm{N})$ ', or 'Force in N'.

- Units are typese upright, not italicized and need a space between the number and unit. Correct: $3 \mathrm{~kg}$. Double incorrect: $3 \mathrm{~kg}$.

- Units, and especially SI base units, are not written out completely anywhere in the text. Incorrect: seconds, sec, kilogram(s), meter(s), Kelvin, Ampère(s), Volt(s), radian(s). Correct: s, $\mathrm{kg}, \mathrm{m}, \mathrm{K}$ (or ${ }^{\circ} \mathrm{C}$ ), $\mathrm{A}, \mathrm{V}$, rad. Unit for weight (' $\mathrm{kg}$ ') is never with a capital $\mathrm{K}$.

- Do not use ' $*$ ' to indicate multiplication. Reserve this operator for convolution. Use no multiplication operator 
between variables, or use ' ' only if needed to avoid ambiguity.

- Do not start sentences with a number, parameter symbol, variable symbol or an equation. It helps to use constructions like 'The stiffness $k$ ' or 'A value of 20...'.

- Equation numbering: discuss with your supervisors if you will number all equations or only equations that you explicitly reference. In MS Word, equation numbering has to be hacked by using invisible tables.

- Make equations part of sentences. Place full stops when a sentence ends after your equation, or a comma when appropriate. Example: 'The relation between weight $x$ and lifespan $y$ is given by the affine relation

$$
y=a x+b
$$

where $a$ and $b$ are...'.

- Do not use (forward) references to equations like you do to figures and tables, because they are parts of sentences. Do not do: 'Equation (1) shows the relation between something and something else.

$$
y=x^{2}
$$

This equation...'. But you can reference them after they have been stated. Example: 'filling in equation (1) in equation (2) shows...', or 'filling in (1) in (2) shows...'.

- Only direct inline equations can use the forward slash ' for division if that improves readability. Example: 'where $\omega_{0}^{2}=k / m$ gives...'. Avoid this type of construction if you can.

- Colloquial use of \pm as to say 'approximately' is incorrect. The \pm symbol indicates to use both the plus and minus. Proper use for uncertainty: 'a value of $3.0 \pm 0.1 \mathrm{~kg}$ ' or two results: $y= \pm \sqrt{x}$. There is no common symbol for 'approximately'.

- Brackets (.), norm lines $\|$.$\| , accolades \{$.$\} , etc. should$ encompass tall equations completely.

$$
\text { Incorrect: }\left(\frac{a}{b}\right),\left\|\frac{y}{\alpha}\right\| \text {, correct: }\left(\frac{a}{b}\right),\left\|\frac{y}{\alpha}\right\| \text {. }
$$

- Align multi-line equations for easy readability. Add line breaks only between separate terms.

- Use the word 'equation' instead of 'formula' (unless you are a chemist).

\section{FiguRES AND TABLES}

\section{Required}

- Reference all figures and tables in the text. If you do not reference it, the figure or table should not be included.

- For figure and table references (e.g. 'see Fig. 3') there can be no line-break between Fig. and the number. This non-line-breaking space can be forced in $\mathrm{LT}_{\mathrm{E}} \mathrm{X}$ with a tilde: Fig. \ref $\{$ figlabel\}, or in MS Word with CTRL+SHIFT+SPACE.

- Figure and table numbers (labels) in the appendices have an appendix letter prefix and you count again from 1. The first appendix figure in appendix $\mathrm{A}$ is Figure A.1, the first in B is Figure B.1, and similarly for tables.
- Figures and tables cannot go beyond page margins, although they can span multiple columns.

- Figures always need a caption placed below the figure. The caption explains the figure and state the key points. Short captions of a few words (e.g. 'The measurement setup') are insufficient and not explanatory. Preferably, the caption font should be smaller than the main document font.

- Tables always need a captions placed above the table. The caption explains the table. Explanations of table contents are place below the table (e.g. abbreviations or statistics). Preferably make the caption and explanation font smaller than the main document font.

- Tables only use horizontal lines at the top and bottom and to separate the header from the content. Avoid the use of vertical lines. See for example this summary ${ }^{11}$ [5] (generally applicable).

- Use vector graphics whenever possible; do not rasterize text. Sufficiently downsample your data before converting to vector graphics.

- Make sure all figures and tables are readable in black and white or grayscale printing and by colorblind people. So, do not (only) color-code information, but use different line styles or thicknesses or grayscale. A colorblindness and grayscale simulator ${ }^{12}$ exists. Or use these color maps $^{13}$ from Okabe and Ito [6].

- All text in figures and tables should be readable at $100 \%$ screen zoom or when printing. Do not use font sizes any smaller than the footnote size of your text. A common mistake is to make a MATLAB or SPSS figure and save it at some random (window) size. When you rescale them to the page/column width you end up with some random font size and awkward aspect ratio.

- Be consistent with font type and font size for text and symbols in figures and tables. A package that helps for figures here is psfrag. You could also make plots directly in LTEX with pgfplots and Tikz. For MATLAB figures, labels and ticks can be set to $\mathrm{T}_{\mathrm{E}} \mathrm{X}$ interpretation. There is also a powerful mat lab $2 t i \mathrm{kz}^{14}$ function.

- When drawing block diagrams (signal flow diagrams), follow IEC notation [7]. Examples: Use circles for summing points, position algebraic signs at the right side of incoming action lines, use dots for branching points, use rectangular blocks. Stick to such formal representation styles.

\section{Writing Advice}

- In a two column document, preferably push the figures and tables to the bottom or top of the column, instead of placing them in the text. This avoids orphan lines of text above, below or between figures or tables that might be missed or look like captions.

- In MS Word, group figures and their captions so they do not separate.

- Figures and tables are both center-aligned horizontally within a column or page. They are preferably not wrapped by text unless unavoidable. This might lead to white space to the left and right for tall figures. 
- In exceptional cases you can rotate the figure or table to make a large full page version.

\section{Punctuation}

Punctuation is used to improve readability and aid understanding by providing suitable pauses between clauses in the text. A few forms of punctuation and their definitions are given below. If unsure about their usage, consult a reliable source before using them in your document.

- Period (.) All sentences must end with a period. This applies to figure and table captions as well as equations that form the end of a sentence. Chapter and (sub)section titles do not end with a period.

- Comma (,) use: commas are severely underused, often abused and heavily misused. Because there is so much to say about commas, look up a decent book on grammar. (Do not forget the Oxford comma!)

- Note that there are four different kinds of dashes: the hyphen '-', the en-dash '-', the em-dash '- ', and the minus sign '-'. They are used in different scenarios:

- hyphen (-): compound single words: red-haired.

- en-dash (-): compound multiple words: pre-World War (to avoid pre-World-War), ranges: pg 1-9.

- em-dash (-): to use for subordinate clauses (NL 'bijzin')

- Colon (:) use: when you announce something, a list, noun or quotation.

- Semi-colon (;) use: to combine independent clauses to avoid choppy separate sentences.

- Quotes: quotes should be opening (" or ") and closing (' or "). In MS Word this is done automatically. In $\mathrm{LT}_{\mathrm{E}} \mathrm{X}$ use one or more ' for opening and ' for closing quotes. Incorrect: "something" ("something" or' ' something' '), correct: "something" ('something'"). Double quotes are often reserved for quotations.

- Apostrophe: the genitive (possessive) always uses apostrophe $+\mathrm{s}$ ('the machine's size'), even when a word ends in ch, $\mathrm{z}$ or $\mathrm{x}$. The only exception is when a word or name already ends in an $s$, then only the apostrophe is used ('planet Mars' orbit'). Apostrophes are not used to indicate the plural form. Incorrect: The car's are moving slowly.

\section{Latin AbBreviations}

Common Latin abbreviations that are used in writing, but should never be used in oral (academic) presentations:

- i.e., stands for id est, which means 'that is'. Use it to make a statement more specific and exclusive. Do not start a sentence with 'I.e.', it should appear after a comma or at the start of a sentence fragment between brackets.

- e.g., stands for exempli gratia, which means 'for example'. Use it to give one or more nonexclusive examples. Do not start a sentence with 'E.g.', it should appear after a comma or at the start of a sentence fragment between brackets.

- et al., stands for et alii, which means 'and colleagues'. The period is required after the 'al'.

\section{Further Writing Resources}

- P.C. Wren, High School English Grammar \& Composition $^{15}$ (ISBN: 935253008X), a good resource on writing in English.

- Mensh and Kording, Ten simple rules for structuring papers [8]. The authors present ten rules to help you communicate the main idea of your paper. They state it will make your writing more efficient and more pleasurable!

- Whiteside et al., Whitesides' Group: Writing a Paper [9]. The authors focus on outline writing and how it also helps you in structuring your research and thinking about your project.

- Rolandi et al., A brief guide to designing effective figures for the scientific paper [10]. The title is quite selfexplanatory.

\section{REFERENCES}

[1] H. Vallery, "Report writing: Guidelines and template," 2020.

[2] C. Andrade, "How to write a good abstract for a scientific paper or conference presentation," Indian journal of psychiatry, vol. 53, no. 2, p. 172, 2011.

[3] Bureau International des Poids et Mesures, "Le Système international d'unités / The International System of Units," SI Brochure, 8th edition, 2006, updated in 2014. [Online]. Available: https: //www.bipm.org/en/publications/si-brochure/

[4] ISO80000-1:2009 Quantities and units - Part 1: General, International Organization for Standardization (ISO) Std. 1, November 2009. [Online]. Available: https://www.iso.org/obp/ui/\#iso:std:iso:80000:-1:ed-1:v1:en

[5] M. Püschel, "Small guide to making nice tables," 2009. [Online]. Available: https://people.inf.ethz.ch/markusp/teaching/guides/guide-tables.pdf

[6] M. Okabe and K. Ito, "How to make figures and presentations that are friendly to color blind people," University of Tokyo, 2002. [Online]. Available: http://jfly.iam.u-tokyo.ac.jp/color/

[7] Electropedia: The World's Online Electrotechnical Vocabulary. Area: 351: Control technology, Section 351-44: Structures of control systems, International Electrotechnical Commission Std. [Online]. Available: http://www.electropedia.org/iev/iev.nsf/index?openform\&part $=351$

[8] B. Mensh and K. Kording, "Ten simple rules for structuring papers," 2017. [Online]. Available: https://journals.plos.org/ploscompbiol/article? id $=10.1371$ /journal.pcbi.1005619

[9] G. M. Whitesides, "Whitesides' group: writing a paper," Advanced Materials, vol. 16, no. 15, pp. 1375-1377, 2004. [Online]. Available: https://onlinelibrary.wiley.com/doi/pdfdirect/10.1002/adma.200400767

[10] M. Rolandi, K. Cheng, and S. Pérez-Kriz, "A brief guide to designing effective figures for the scientific paper," Advanced Materials, vol. 23, no. 38, pp. 4343-4346, 2011. [Online]. Available: https://onlinelibrary.wiley.com/doi/abs/10.1002/adma.201102518

\section{EXTERNAL LINKS}

${ }^{1}$ https://bitbucket.org/ctw-bw/thesis-templates

${ }^{2}$ http://people.utwente.nl

${ }^{3} \mathrm{https}$ ://support.microsoft.com/en-us/office/add-different-page-numbersor-number-formats- to-different-sections-bb4da2bd-1597-4b0c-9e91$620615 \mathrm{ed} 8 \mathrm{c} 05$

${ }^{4}$ https://www.overleaf.com/learn/latex/Page_numbering

${ }^{5}$ https://www.merriam-webster.com/

${ }^{6} \mathrm{http}: / /$ essay.utwente.nl

${ }^{7}$ https://www.mendeley.com/reference-management/reference-manager

${ }^{8}$ https://oeis.org/wiki/List_of_LaTeX_mathematical_symbols

${ }^{9} \mathrm{https}: / /$ pages.mtu.edu/ $\sim$ bco/cm $416 /$ EquationEditor_main.pdf

${ }^{10} \mathrm{https}: / /$ detexify.kirelabs.org/classify.html

${ }^{11} \mathrm{https}: / /$ people.inf.ethz.ch/markusp/teaching/guides/guide-tables.pdf

${ }^{12} \mathrm{https} / / / \mathrm{www}$. color-blindness.com/coblis-color-blindness-simulator/

${ }^{13} \mathrm{http}$ ://jfly.iam.u-tokyo.ac.jp/color/image/pallete.jpg

${ }^{14} \mathrm{https}: / /$ github.com/matlab2tikz/matlab2tikz

$15 \mathrm{https} / / / \mathrm{www}$.amazon.nl/HIGH-SCHOOL-ENGLISH-GRAMMARCOMPOSI/dp/935253008X 